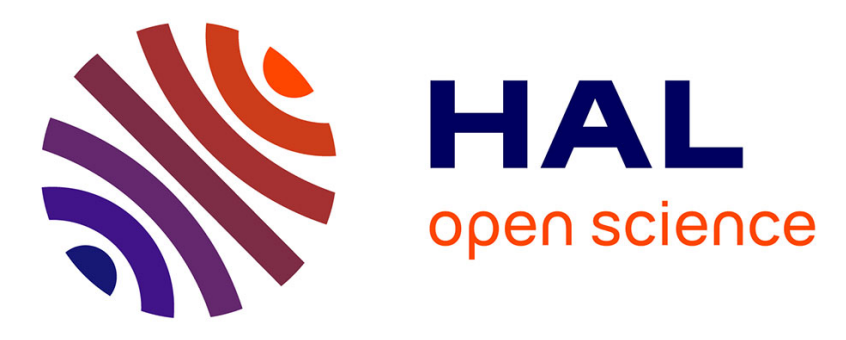

\title{
Dynamic Effect of an Active Deformable Flat Plate on Its Turbulent Flow
}

\author{
T. Deloze, Yannick Hoarau, M. Chinaud, Johannes Scheller, E. Deri, \\ Jean-François Rouchon, Eric Duhayon, Marianna Braza
}

\section{- To cite this version:}

T. Deloze, Yannick Hoarau, M. Chinaud, Johannes Scheller, E. Deri, et al.. Dynamic Effect of an Active Deformable Flat Plate on Its Turbulent Flow. Marianna Braza; Alessandro Bottaro; Mark Thompson. Advances in Fluid-Structure Interaction, 133, Springer International Publishing, pp.309316, 2016, 978-3-319-27384-6. 10.1007/978-3-319-27386-0_19 . hal-03144886

\author{
HAL Id: hal-03144886 \\ https://hal.science/hal-03144886
}

Submitted on 17 Feb 2021

HAL is a multi-disciplinary open access archive for the deposit and dissemination of scientific research documents, whether they are published or not. The documents may come from teaching and research institutions in France or abroad, or from public or private research centers.
L'archive ouverte pluridisciplinaire HAL, est destinée au dépôt et à la diffusion de documents scientifiques de niveau recherche, publiés ou non, émanant des établissements d'enseignement et de recherche français ou étrangers, des laboratoires publics ou privés. 


\title{
DYNAMIC EFFECT OF AN ACTIVE DEFORMABLE FLAT PLATE ON ITS TURBULENT FLOW
}

\author{
T. Deloze ${ }^{a}$, Y. Hoarau ${ }^{b}$, M. Chinaud $^{c}$, J. Scheller ${ }^{c}$, E. Deri ${ }^{a}$, \\ J.F. Rouchon ${ }^{c}$, E. Duhayon ${ }^{c}$ and M. Braza ${ }^{a}$ \\ ${ }^{a}$ Institut de Mécanique des Fluides de Toulouse, Unité Mixte C.N.R.S.-I.N.P.T. 5502, Toulouse, France \\ ${ }^{b}$ Icube, Université de Strasbourg - C.N.R.S., Strasbourg, France \\ ${ }^{c}$ LAPLACE, CNRS - INPT - UPS, Toulouse, France \\ tdeloze@imft.fr, marianna.braza@imft.fr, hoarau@unistra.fr
}

\begin{abstract}
.
This paper addresses the topic of the influence of the profile of a plate on a turbulent flow. The study is based on numerical results obtained by URANS and hybrid (DDES) turbulence modeling and compared with experimental results. The flat plate has an aspect ratio of 20 (length over thickness) and the edges are not smoothed. The plate includes Shape Memory Alloys (SAM), which allows it to arch under the effect of an electric voltage. The deformation by the material corresponds to a beam deformation and is implemented in the numerical simulation according to this definition. The Reynolds number is $R e=200.000$, which led to a turbulent flow with a Von Karman vortex shedding and Kelvin Helmotz instabilities in the shear layer. The simulations are performed with several turbulence models: k- $\epsilon$-OES, k- $\omega$-BSL-OES, k- $\omega$-DDES-BSL-OES, k$\omega$-DDES-SST-Menter. These models are used in a first step to determine the three-dimensional flow around the plate plane inclined by 10 degrees. The behavior of the flow are found similar between experiment and numerical simulations. The purpose of the curve shape by deformation is then compared to a linear form by rotation of the plate. The curved shape provides the same lift coefficient with less required deformation and thus with a lower drag coefficient and also a lower frequency. The dynamic deformation is slow compared to the freestream velocity, it weakly affects the coefficients and frequencies compared to linear shape.
\end{abstract}

Key words: turbulence, flat plate, shape memory alloys, deformation.

\section{Introduction}

The interest for micro and nano air vehicles is increasing in recent years. Their development open some problems in aerodynamics, as the control, with demanding constraints, such as weight. Meanwhile during the last ten years, many morphing materials have been developed and studied. These materials have opened new opportunities in fluid mechanic applications as morphing wing. The shape of an airfoil can be locally modified by this morphing technology. In this work, we focus on the dynamic effect of the deformation imposed by morphing material on a turbulent flow. The final goal is an optimised integration into the flight controls of micro airplane. 
The numerical present study is based on the turbulent flow around a flat plate at incidence with untapered edges. The flat plate is equipped with Shape Memory Alloys (SAM) that allow dynamic morphing by electric power. The deformation is an camber of the plate with uniform deformation in the span direction. The first part of the study aims at the analysis of the 3-dimensional wake behind a flat plate at incidence with an attention to capture the vortex shedding and the shear layer with numerical results. This focus is motivated by the harmful effect of the both phenomena on the aerodynamic performance and on the noise production. Then the influence of the shape is examined in 2-dimensional simulations, to determine the effect of the curve shape profiled by the SAM equipment compared to the linear shape. At the last, the dynamic effect on the flow is discussed with 2-dimensional simulations.

\section{Configuration}

The configuration is defined by a previous experimental study performed at the Institut de Mécaniques des Fluides de Toulouse (E. Deri et al. [2]). The experiment involves a flat plate (cf. figure 1) with an aspect ratio of 20 (width $(l)$ over thickness $(h)$ ), an angle of incident of 10 degrees and a turbulent flow characterized by the Reynolds number of 200000 based on the freestream flow velocity $(U)$, the width of the plate and the kinematic viscosity $(\nu)$.
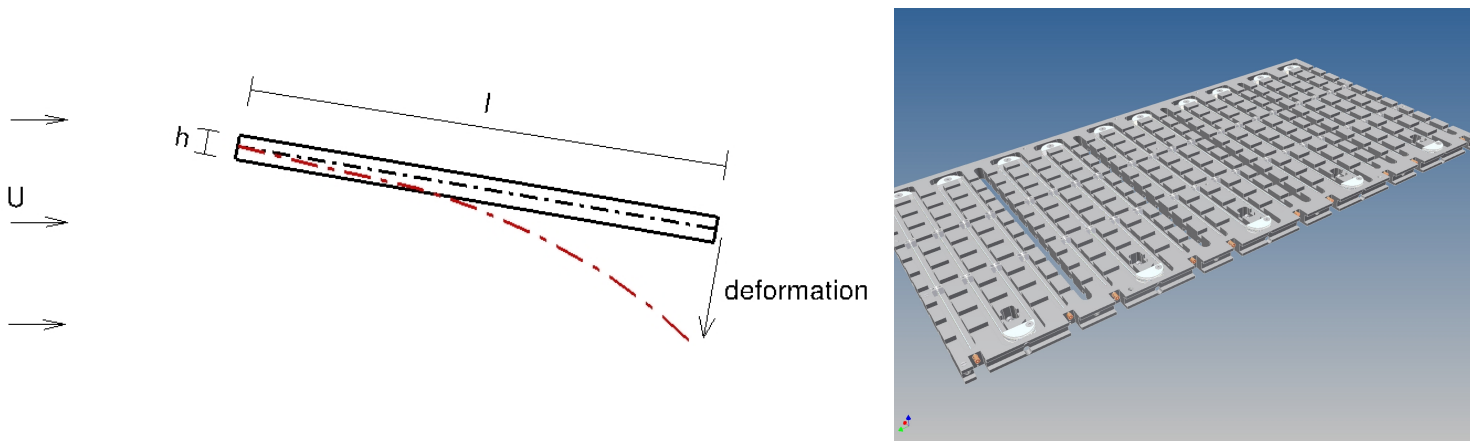

Figure 1: Configuration 2d of the flat plate and the deformation (left) and the 3D frame of the plate (right)

The experiments (cf. figure 2) have been carried out in the S4 wind tunnel and the results are obtained by 3D tomographic PIV (E. Deri et al. 2012 [2]) for a rigid plate (without deformation). The Proper Orthogonal Decomposition (POD) was performed and the higher modes show the Von Karman pattern. The tomographic PIV results are obtained with a slow speed camera and do not allow a time correlation of the velocity. To complete this study, a second round of wind tunnel testing is conducted with a high speed camera and for the dynamic deformation of the plate. These results (cf. figure 5, right) are used to determine the velocity with time resolution (time resolved PIV). The images obtained were also used to characterize the displacement of the trailing edge of the plate, which is deformed by excitation of the SAM material (cf. figure 6). 

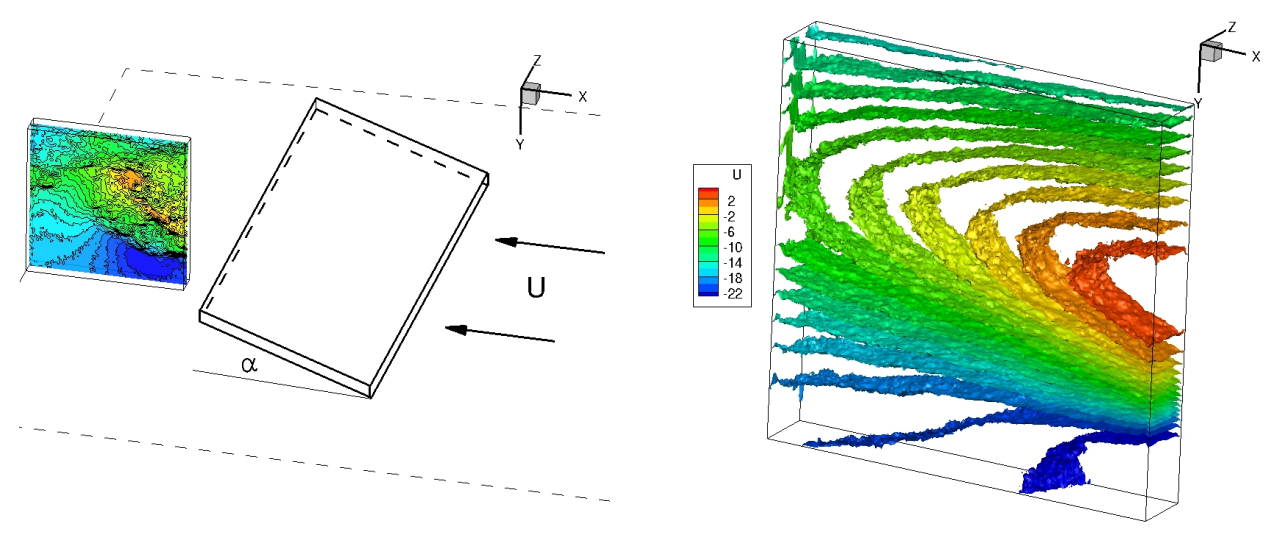

Figure 2: Description of the wind tunnel experiment, E. Deri et al. 2012 (left) and representation of the $3 \mathrm{D}$ isovalues of the average of the streamwise velocity (right)

\section{Results}

The numerical simulations are performed with NSMB software $[5,7,6]$ based on the resolution of the Navier-Stokes equation (LU-SGS scheme for the implicit time discretisation and central scheme with Jameson dissipation for the space integration) and the OES turbulence modeling approach developed in the IFS2T team (Braza et al. [1]). This approach is based on the splitting of the energy spectrum into two parts, the first regrouping the organised coherent motion (resolved turbulence) and the second, the chaotic random turbulence (modeled part). This OES modeling achieves to capture the thin shear-layers for the flow with coherent structures. The flow around the inclined plate hold the quasi-periodic structures (Von-Karman vortex shedding) and the shear-layer, appropriate for OES modeling.

\subsection{D simulations of the static flat plate}

The first set of results is related to the static case (initial linear shape with an angle of attack equal to 10 degrees). These results provide a validation of the numerical method and shows good accuracy in the prediction of the flow with a comparison of time-averaged velocity of the flow. The Von-Karman vortex shedding appears in the wake with the Kelvin-Helmotz vortices in the shear layer (cf. figure 5).

The comparison of the turbulence models (k- $\epsilon, \mathrm{k}-\omega$-BSL-OES, k- $\omega$-DDES-BSL-OES, $\mathrm{k}-\omega$-DDES-SST-Menter) was conducted. The evolution of the coefficients are similar except for the $\mathrm{k}-\epsilon$-OES (see figure 4). The $\mathrm{k}-\omega$-BSL-OES model shows a good agreement with the experimental results (tomographic PIV) for the comparison of the time average of streamwise velocity (figure 4). A dominant period appears for all the cases around $S t=0.72$. The frequency is induced by the Von Karman vortex shedding.

The DDES approach does not radically change the mean values of the coefficients although instantaneous velocity are different (see figure 3). Figure 3 (right) shows the presence of vortices arising in the leading edge and deformed to horseshoe vortices. The Von Karman vortex shedding in the wake is clearly defined for none hybrid model and this coherent structures disappear in the wake for the visualisation of 

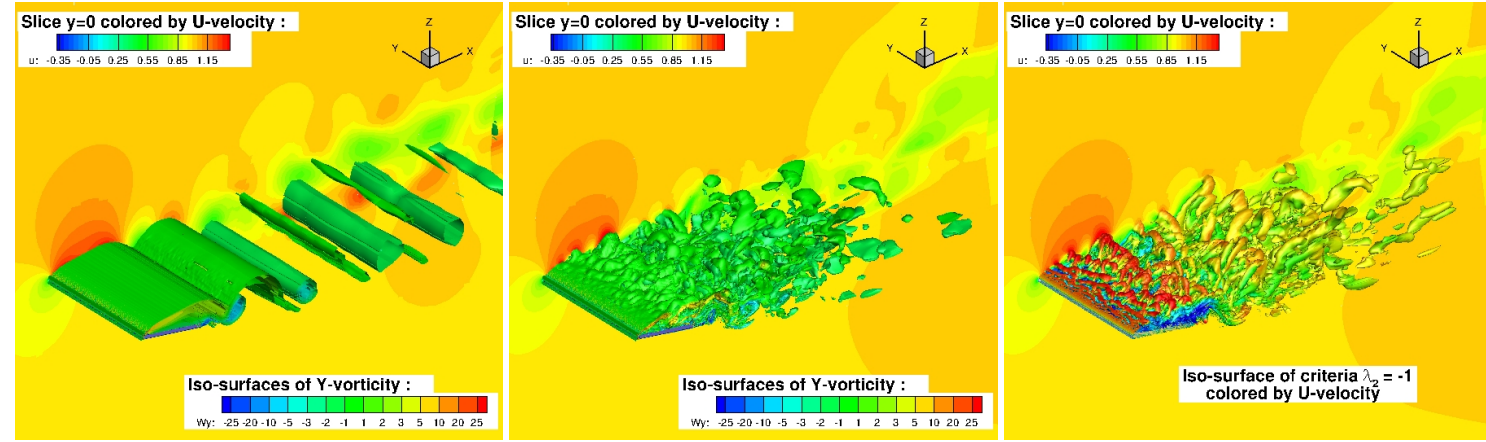

Figure 3: Instatenious field of the isovalues of Y-vorticity for $\mathrm{k}-\epsilon-O E S$ (left), k- $\omega$-DDESSST-Menter (middle) and the isovalue -1 of the criteria $\lambda_{2}$ for $\mathrm{k}-\omega$-BSL-OES (right)
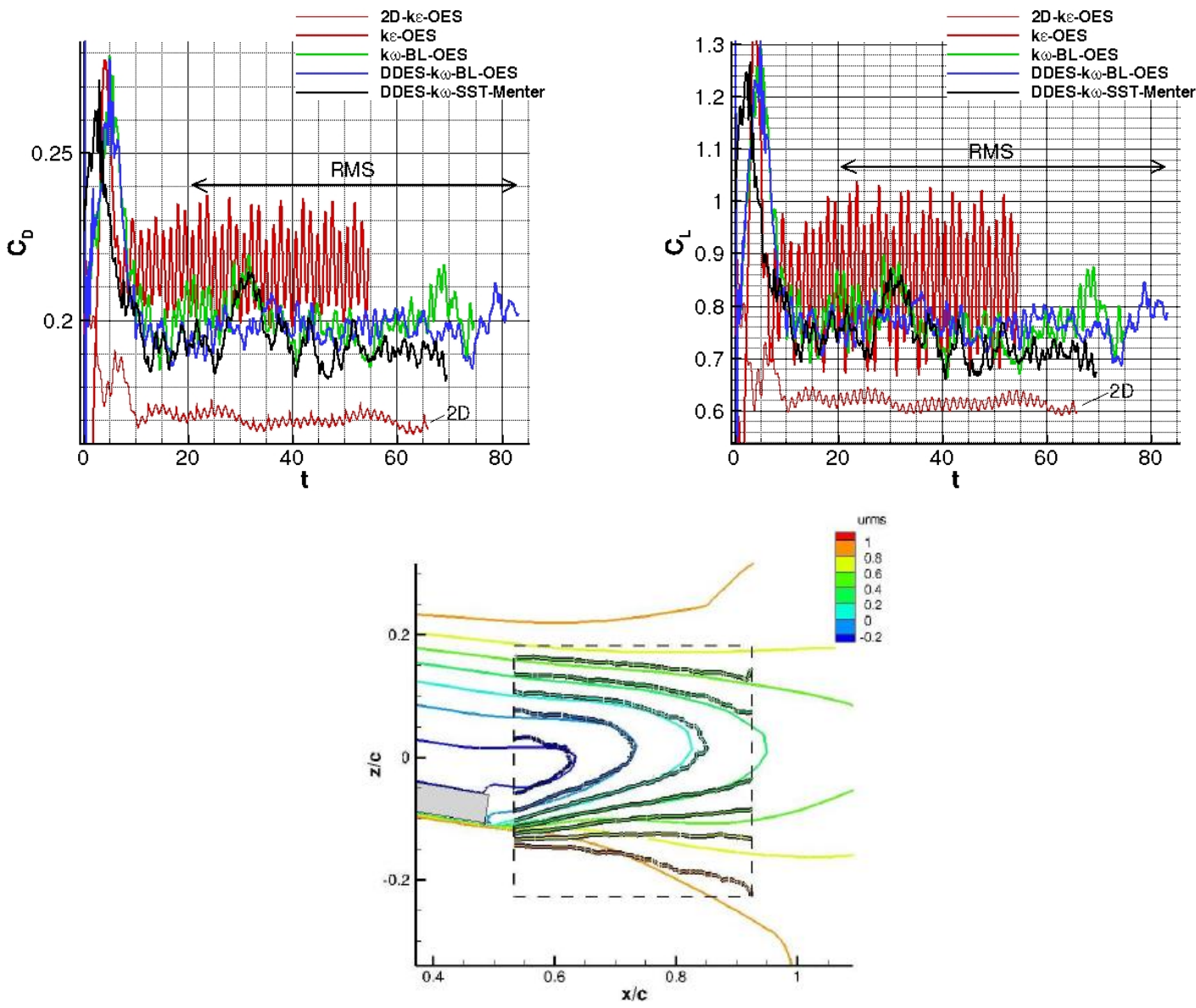

Figure 4: Temporal evolution of the drag and the lift coefficients (respectively left and middle figures) and the comparison of the temporal average of the streamwise velocity of the k- $\omega$-BSL-OES versus the TOMO results

DDES model.

The representation of the streamwise velocity in the plane perpendicular to the plate present the same flow characteristics that appear in the TR-PIV measurements (figure 5), with the Kelvin Helmotz vortices in the shear layer. 

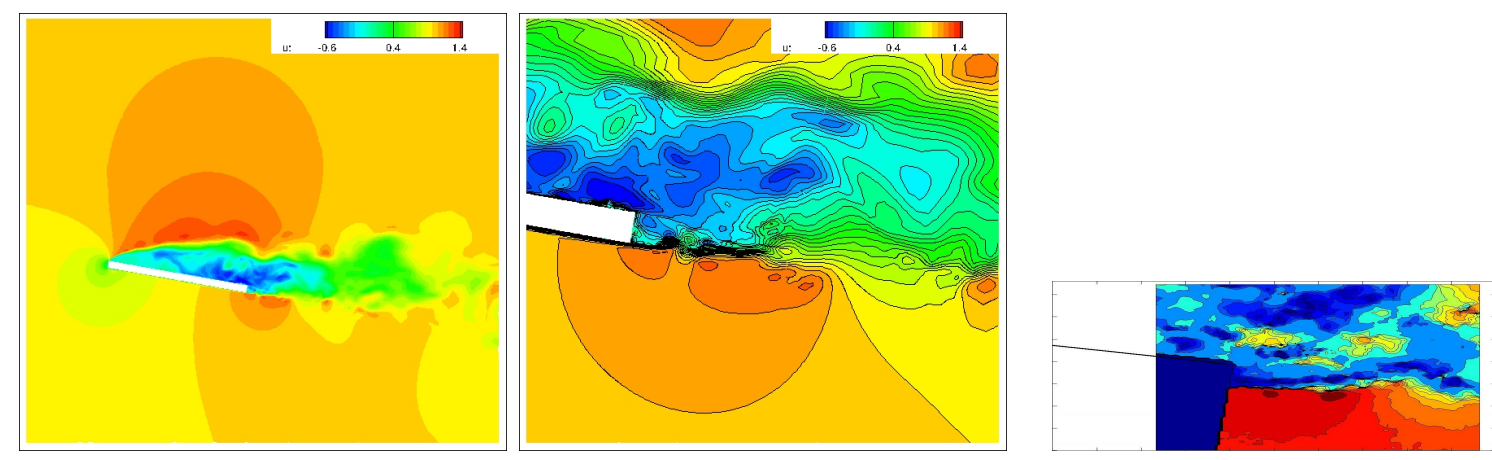

Figure 5: In the plane perpendicular to the plate, isovalues of the streamwise velocity (left) and a zoom on the trailing edge (middle) for k- $\omega$-DDES-SST-Menter and the same representation for the TR-PIV (right)

\subsection{D static simulations of the several deformations}

In the second step, the 2D simulations are performed for five forms of the plate during its deformation (position 1 is close to its initial position, and position 5 is close to its maximum deformation). The figure 6 shows the deformation. This deformation is obtained by activating the SAM material. To get closer to the experience, the position of the trailing edge is determined with the TR-PIV results and imposed in the numerical simulations. The deformation of the plate then follows the law of deformation of a beam. These results are compared to linear plate shape (figure 6).
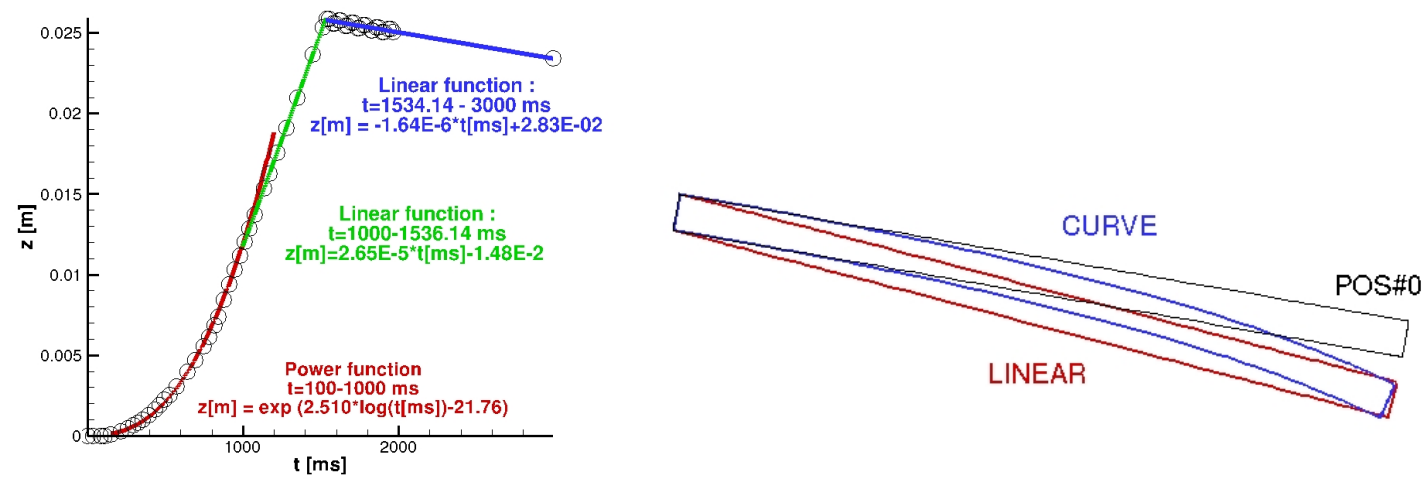

Figure 6: Evolution of the position of the trailing edge (left) and description of the curve and linear shapes of the plate for the position $\# 5$

The frequencies calculated with the lift coefficient, characterizing vortex shedding, are close between curve and linear shapes. This result is consistent with the results of Fage [3], indicating that the frequency depends on the length of the projection of the plate in the cross flow direction. The coefficient of lift and drag have a much more pronounced difference. The curved shape provides higher values of the both coefficients (cf. figure 7 for the lift coefficient). For a given coefficient, the value of the required deformation is lower in the case of the curve shape. For example, for $\operatorname{rms}\left(C_{L}\right)=0.5$, the curve shape reaches to a position close to 3 , while the linear form near the position 4 . Thus for the same lift coefficient, the frequency will be 
higher, because the inclination of the plate will be lower. The same goes for the drag coefficient. The comparison of the instantaneous streamwise velocity between the curve and linear shape plate (figure 8) presents closed results.
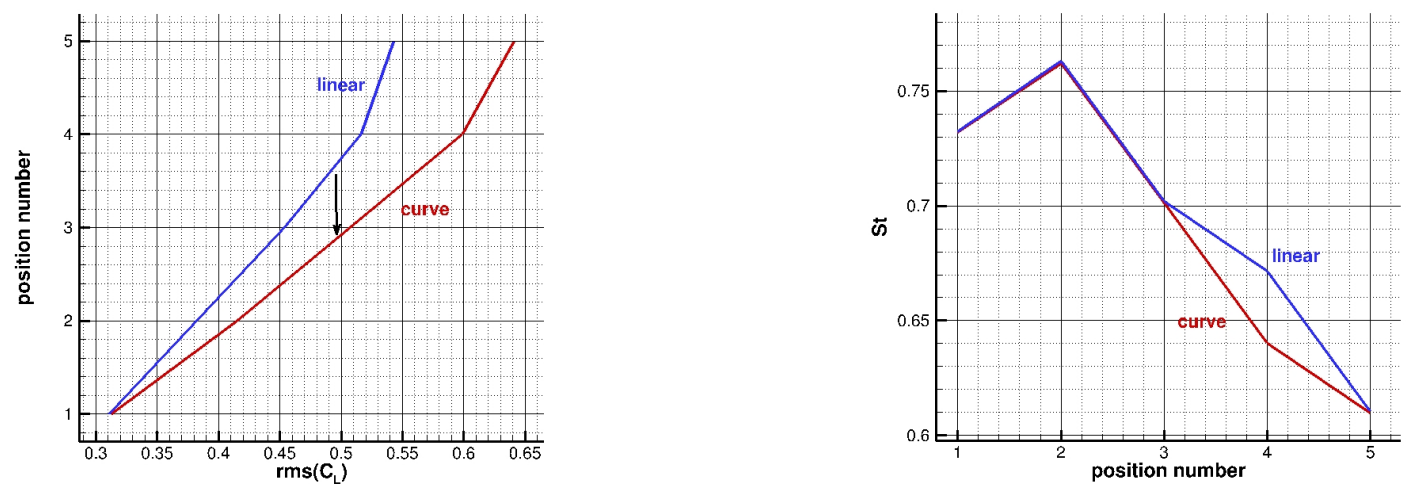

Figure 7: Deformation versus the root mean square of the lift coefficient (left) and versus the Strouhal number (right) for the two shapes
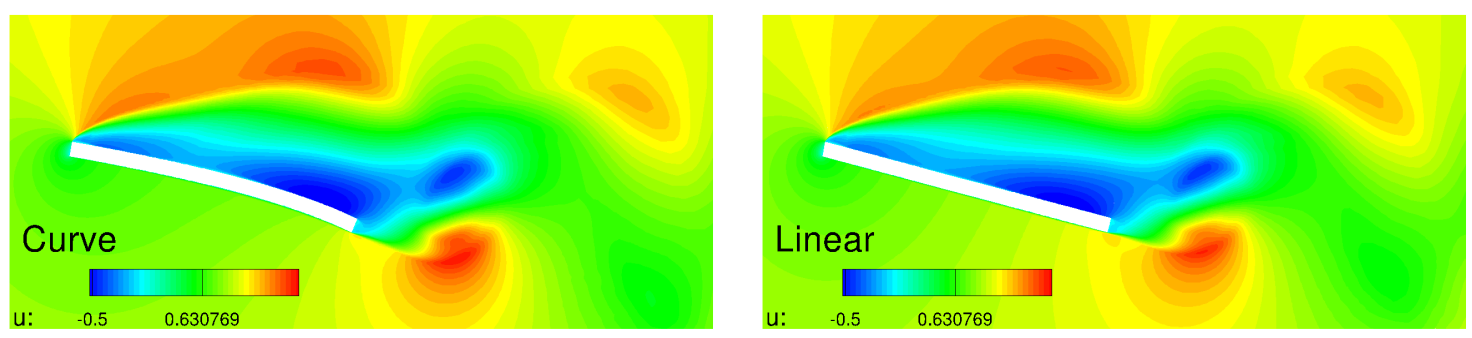

Figure 8: Streamwise velocity for the position $\# 5$ for curve shape (left) and linear shape (right)

\subsection{D dynamic simulations}

The final step is the determination of the influence of the dynamics of the deformation. In the experiment, the deformation rate of the plateis about $1 / 1000^{\text {th }}$ of the freestream velocity which means that compared to the period of Von Karman instability, the maximum deformation is reached after about 50 Von Karman vortex shedding. The deformation is slow relative to the flow dynamic. This can explain the small -or negligible- difference between the forces appearing between static and dynamic cases. Thus, the dynamics can be considered as a quasi-static state succession. We have computed five deformation speed : $x 0.5, x 1, x 2, x 4$ and $x 8$ and at each time step the grid is updated through a parallel Volume Spline Interpolation proposed by Spekreijse et al [4]. Figure 9 compare the time evolution of the aerodynamics coefficients regarding the different deformation velocities and we can that this deformation velocity has no influence on the final state of the system. The final evolution of the flow is the same, the drag and lift are higher than the static case and the strouhal number is lower. 

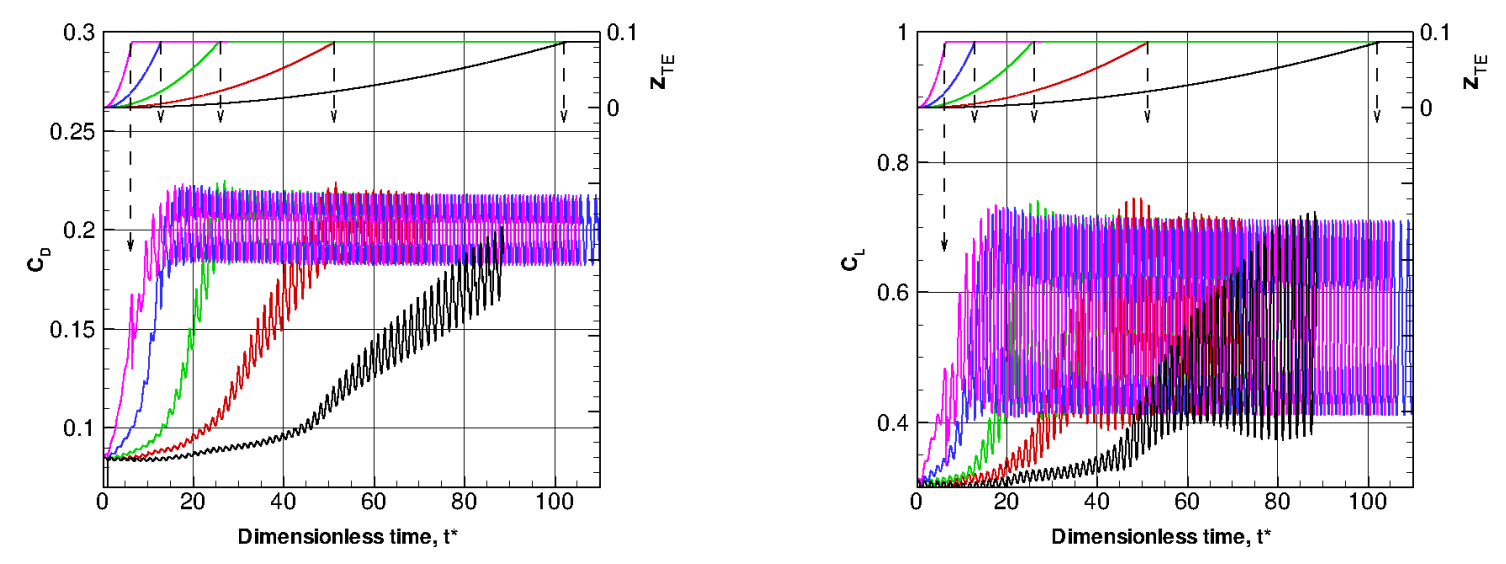

Figure 9: Time evolution of the aerodynamics coefficients for the different deformation velocities : black (x0.5), red (x1), green $(\mathrm{x} 2)$, ble $(\mathrm{x} 4)$ and purple $(\mathrm{x} 8)$

\section{Conclusions}

This work has shown a good prediction of the 3D flow with URANS and hybrid turbulent models for flow around a fixed flat plate. The 2D study of the influence of the profile shape demonstrates that curved shape reached lift coefficient with less required deformation. Dynamic simulation of deformation shows that the deformation is too slow to strongly influence the vortex structures and the flow can be considered as a succession of static states. The outline is a $3 \mathrm{D}$ dynamic simulation of the deformation.

\section{Acknowledgements}

The present work was supported by the Foundation STAE-RTRA, research programm EMMAV. The authors would like to acknowledge the following high performance computing centers for supporting this work by providing scientific support and access to computing resources : CINES/GENCI, PRACE and the Direction Informatique of the University of Strasbourg.

\section{References}

[1] M. Braza, R. Perrin, and Y. Hoarau. Turbulence properties in the cylinder wake at high reynolds number. J. of Fluids and Structures, 22, 2006.

[2] E. Deri, M. Braza, E. Cid, S. Cazin, D. Michaelis, and C. Degouet. Investigation of the threedimensional turbulent near-wake structure past a flat plate by tomographic piv at high reynolds number. J. of Fluids and Structures, 2006.

[3] A. Fage and F. C. Johansen. On the flow of air behind an inclined flat plate of infinite span. Proceedings of the Royal Society of London. Series A, 116, 1927.

[4] S. P. Spekreijse, B. B. Prananta, and J. C. Kok. A simple, robust and fast algorithm to compute deformations of multi-block structured grids. Technical report, National Aerospace Laboratory NLR, 2002. 
[5] J. Vos, P. Leyland, P. Lindberg, V. Kemenade, C. Gacherieu, N. Duquesne, P. Lötstedt, C. Weber, and A. Ytterström. NSMB Handbook version 4.0. 1997.

[6] J. Vos, A. Rizzi, A. Corjon, E. Chaput, and E. Soinne. Recent Advances in aerodynamics inside the NSMB (Navier-Stokes Multiblock) Consortium. AIAA paper, 98-0225, 1998.

[7] J. Vos, A. Rizzi, D. Darracq, and E. Hirschel. Navier-Stokes solvers in European aircraft design. Progress in Aerospace Sciences, 38:601-697, 2002. 